PROCEEDINGS OF THE

AMERICAN MATHEMATICAL SOCIETY

Volume 129, Number 12, Pages 3749-3752

S 0002-9939(01)06027-0

Article electronically published on May 7, 2001

\title{
CHERN NUMBERS OF ALMOST COMPLEX MANIFOLDS
}

\author{
HANSJÖRG GEIGES
}

(Communicated by Ralph Cohen)

\begin{abstract}
It is shown that any system of numbers that can be realised as the system of Chern numbers of an almost complex manifold of dimension $2 n, n \geq 2$, can also be realised in this way by a connected almost complex manifold. This answers an old question posed by Hirzebruch.
\end{abstract}

Let $\pi(n)$ denote the number of partitions of the natural number $n$. A theorem of Milnor (cf. [5]) states that a system of $\pi(n)$ numbers can be realised as the system of Chern numbers of an almost complex manifold of (real) dimension $2 n$ if and only if it can be realised in this way by some algebraic manifold of (complex) dimension $n$ belonging to some class $\mathcal{A}$. (Manifolds are understood to be oriented, differentiable, and compact without boundary.) The class $\mathcal{A}$ is generated (under cartesian product and disjoint union) by the complex projective spaces, the hypersurfaces $H_{(r, t)}$ of double degree $(1,1)$ in $\mathbb{C} P^{r} \times \mathbb{C} P^{s}$ with $r, s>1$, and certain algebraic manifolds which realise the negative of the Chern numbers of the manifolds already listed.

Thus, at least in principle, it is known which systems of $\pi(n)$ numbers can be realised as the Chern numbers of a $2 n$-dimensional almost complex manifold. In low dimensions, a complete set of restrictions is given as follows (cf. [5]):

$$
\begin{aligned}
& n=1: \quad c_{1} \equiv 0 \bmod 2, \\
& n=2: \quad c_{1}^{2}+c_{2} \equiv 0 \bmod 12 \text {, } \\
& n=3: \quad c_{1} c_{2} \equiv 0 \bmod 24, c_{1}^{3} \equiv c_{3} \equiv 0 \bmod 2 \text {, } \\
& n=4: \quad-c_{1}^{4}+4 c_{1}^{2} c_{2}+c_{1} c_{3}+3 c_{2}^{2}-c_{4} \equiv 0 \bmod 720 \text {, } \\
& 2 c_{1}^{4}+c_{1}^{2} c_{2} \equiv 0 \bmod 12, c_{1} c_{3}-2 c_{4} \equiv 0 \bmod 4 .
\end{aligned}
$$

In [5] Hirzebruch raised the question whether a system of $\pi(n)$ numbers satisfying the necessary restrictions can be realised as the system of Chern numbers of a connected almost complex manifold of dimension $2 n$, and speculated that the connectedness assumption might impose additional inequalities between the Chern numbers.

If the question is asked for complex or algebraic manifolds, there are indeed additional restrictions on the Chern numbers in the form of inequalities, as was first shown by Van de Ven [13 for complex dimension 2 (cf. [2]). In that paper Van de Ven also proved that no additional restrictions occur for connected almost complex manifolds of real dimension 4.

Received by the editors May 2, 2000.

2000 Mathematics Subject Classification. Primary 57R20, 32Q60.

(C)2001 American Mathematical Society 
In 11 it was shown - the published argument actually being due to Hirzebruch - that in dimension $2 n=6$, too, connectedness does not impose any additional restrictions on the Chern numbers of almost complex manifolds besides those listed above. However, the argument presented there seemed to suggest that this might be special to dimension 6 , since it relied on the fact that the connected sum of two almost complex manifolds of dimension 6 carries again an almost complex structure, which fails, in general, in dimensions other than 2 or 6 ; cf. [1, 8].

In the present note we give a short argument that, nevertheless, this statement about Chern numbers remains true in all dimensions.

Proposition 1. A system of $\pi(n)$ numbers that can be realised as the system of Chern numbers of an almost complex manifold of dimension $2 n, n \geq 2$, can also be realised in this way by a connected almost complex manifold.

This is obviously false for $n=1$. Here the existence of an (almost) complex structure is equivalent with orientability, and the first Chern number equals the Euler characteristic. Hence any even number can be realised by a disconnected surface, but only even numbers $\leq 2$ can be realised by a connected surface.

Proposition 1 is an immediate consequence of the following lemma.

Lemma 2. Let $M_{1}, \ldots, M_{k}$ be connected almost complex manifolds of dimension $2 n$, $n \geq 2$. Then the connected sum

$$
W=M_{1} \# \cdots \# M_{k} \#(k-1) S^{2} \times S^{2 n-2}
$$

admits an almost complex structure which coincides with the given almost complex structures along the $(2 n-1)$-skeleta of the $M_{j}$ and has trivial Chern class on the $(2 n-1)$-skeleta of the $S^{2} \times S^{2 n-2}$ summands. In particular, with respect to the natural splitting

$$
H^{*}(W)=H^{*}\left(M_{1}\right) \oplus \cdots \oplus H^{*}\left(M_{k}\right) \oplus(k-1) H^{*}\left(S^{2} \times S^{2 n-2}\right)
$$

for $0<*<2 n$ we have

$$
c_{i}(W)=\left(c_{i}\left(M_{1}\right), \ldots, c_{i}\left(M_{k}\right), 0, \ldots, 0\right) \text { for } 1 \leq i<n,
$$

and for the Chern numbers $c_{n}$ we have

$$
c_{n}(W)=c_{n}\left(M_{1}\right)+\cdots+c_{n}\left(M_{k}\right) .
$$

So all the Chern numbers $c_{I}, I \in \pi(n)$, satisfy

$$
c_{I}(W)=c_{I}\left(M_{1}\right)+\cdots+c_{I}\left(M_{k}\right)=c_{I}\left(M_{1} \sqcup \cdots \sqcup M_{k}\right),
$$

with $\sqcup$ denoting disjoint union.

Proof. We use the results and conventions of [8]; cf. [10]. Let $M$ be a $2 n$-dimensional manifold, and let $J$ be an almost complex structure on $M-D^{2 n}$ for some embedded disc $D^{2 n}$. Write

$$
\mathfrak{o}(M, J) \in H^{2 n}\left(M ; \pi_{2 n-1}\left(\mathrm{SO}_{2 n} / \mathrm{U}_{n}\right)\right)
$$

for the obstruction to extending $J$ as an almost complex structure over $M$.

Then we have the following statements from 8 :

(i) $\mathfrak{o}\left(S^{2 n}, J\right)$ is independent of $J$ and will be written as $\mathfrak{o}\left(S^{2 n}\right)$. 
(ii) Almost complex structures $J$ on $M-D^{2 n}$ and $J^{\prime}$ on $M^{\prime}-D^{2 n}$ give rise to a natural almost complex structure $J+J^{\prime}$ on $M \# M^{\prime}-D^{2 n}$ (which coincides with $J$ resp. $J^{\prime}$ along the $(2 n-1)$-skeleton of $\left.M \# M^{\prime}\right)$ such that

$$
\mathfrak{o}\left(M \# M^{\prime}, J+J^{\prime}\right)=\mathfrak{o}(M, J)+\mathfrak{o}\left(M^{\prime}, J^{\prime}\right)-\mathfrak{o}\left(S^{2 n}\right) .
$$

(iii) Let $J$ be an almost complex structure on $M-D^{2 n}$ that extends over $M$ as a stable almost complex structure $\widetilde{J}$. Then

$$
\mathfrak{o}(M, J)=\frac{1}{2}\left(\chi(M)-c_{n}(\widetilde{J})\right) \mathfrak{o}\left(S^{2 n}\right),
$$

where $\chi(M)$ denotes the Euler characteristic of $M$.

Write $J_{j}$ for the given almost complex structures on $M_{j}, j=1, \ldots, k$. Then clearly $\mathfrak{o}\left(M_{j}, J_{j}\right)=0$. The manifold $S^{2} \times S^{2 n-2}$ is stably parallelisable, so we can find a stable almost complex structure $\widetilde{J_{0}}$ on $S^{2} \times S^{2 n-2}$ with total Chern class $c\left(\widetilde{J_{0}}\right)=1$. Since the coefficient groups $\pi_{r}\left(\mathrm{SO}_{2 n} / \mathrm{U}_{n}\right)$ for the obstructions to an almost complex structure are stable for $r<2 n-1$ (see [9]) this stable almost complex structure induces an almost complex structure $J_{0}$ on $S^{2} \times S^{2 n-2}-D^{2 n}$.

From (iii) we get

$$
\mathfrak{o}\left(S^{2} \times S^{2 n-2}, J_{0}\right)=2 \mathfrak{o}\left(S^{2 n}\right) .
$$

Hence, from (ii),

$$
\begin{aligned}
\mathfrak{o} & \left(M_{1} \# \cdots \# M_{k} \#(k-1) S^{2} \times S^{2 n-2}, J_{1}+\cdots+J_{k}+(k-1) J_{0}\right) \\
\quad & =\sum_{j=1}^{k} \mathfrak{o}\left(M_{j}, J_{j}\right)+(k-1) \mathfrak{o}\left(S^{2} \times S^{2 n-2}, J_{0}\right)-(2 k-2) \mathfrak{o}\left(S^{2 n}\right) \\
& =0
\end{aligned}
$$

This proves the existence of an almost complex structure $J$ on $W$ for which $c_{i}(W)$, $1 \leq i<n$, is as claimed in the lemma. The Chern number $c_{n}$ coincides with the Euler number, and for the connected sum of even-dimensional manifolds we have

$$
\chi\left(M \# M^{\prime}\right)=\chi(M)+\chi\left(M^{\prime}\right)-2 .
$$

So we get

$$
\begin{aligned}
c_{n}(W) & =\sum_{j=1}^{k} c_{n}\left(M_{j}\right)+(k-1) \chi\left(S^{2} \times S^{2 n-2}\right)-2(2 k-2) \\
& =\sum_{j=1}^{k} c_{n}\left(M_{j}\right) .
\end{aligned}
$$

This proves the lemma.

Remarks. (1) In [12] Ray had proved the analogue of Proposition 1 on the level of stable almost complex structures. A further related result has recently been obtained by Bryan Johnston [7]. He shows that in complex dimension $n \geq 2$ all possible values of the Milnor numbers $s_{n}$ (cf. 66, p. 112]) can be realised by connected algebraic manifolds. In particular, this yields a system of generators for the complex cobordism ring consisting of such connected algebraic manifolds.

(2) The question addressed in this note can also be asked for symplectic manifolds. Concerning (real) dimension 4, a considerable amount of information, but not quite a complete answer, can be found in [3]. In dimension 6, any triple of 
Chern numbers that can be realised by an almost complex manifold can also be realised by a connected symplectic manifold; see [4].

\section{ACKNOWLEDGEMENTS}

I am grateful to Prof. F. Hirzebruch for drawing my attention to the work of Bryan Johnston. I also thank B. Johnston for pointing out Ray's result to me.

\section{REFERENCES}

1. M. Audin, Exemples de variétés presque complexes, Enseign. Math. (2) 37 (1991), 175-190. MR 92g:53024

2. W. Barth, C. Peters, and A. Van de Ven, Compact Complex Surfaces, Ergeb. Math. Grenzgeb. (3) vol. 4, Springer, Berlin, 1984. MR 86c:32026

3. R.E. Gompf, A new construction of symplectic manifolds, Ann. of Math. (2) 142 (1995), 527-595. MR 96j:57025

4. M. Halic, On the geography of symplectic 6-manifolds, Manuscripta Math. 99 (1999), 371-381. MR 2000f:57027

5. F. Hirzebruch, Komplexe Mannigfaltigkeiten, Proc. Internat. Congress Math. 1958, Cambridge University Press, 1960, pp. 119-136. MR 23A:1055

6. _ Topological Methods in Algebraic Geometry, Classics Math., Springer, Berlin, 1995. MR 96c:57002

7. B. Johnston, The values of the Milnor genus on smooth projective connected complex varieties, preprint, University of Michigan.

8. P.J. Kahn, Obstructions to extending almost X-structures, Illinois J. Math. 13 (1969), 336357. MR 41:2684

9. W.S. Massey, Obstructions to the existence of almost complex structures, Bull. Amer. Math. Soc. 67 (1961), 559-564. MR 24A:2971

10. S. Müller and H. Geiges, Almost complex structures on 8-manifolds, Enseign. Math. (2) 46 (2000), 95-107. CMP 2000:15

11. Ch. Okonek and A. Van de Ven, Cubic forms and complex 3-folds, Enseign. Math. (2) 41 (1995), 297-333. MR 97b:32035

12. N. Ray, On a construction in bordism theory, Proc. Edinburgh Math. Soc. (2) 29 (1986), 413-422. MR 88a:57065

13. A. Van de Ven, On the Chern numbers of certain complex and almost complex manifolds, Proc. Nat. Acad. Sci. U.S.A. 55 (1966), 1624-1627. MR 33:6651

Mathematisch Instituut, Universiteit Leiden, Postbus 9512, 2300 RA Leiden, The Netherlands

E-mail address: geiges@math.leidenuniv.nl 